\title{
Report of a deadly case of potter syndrome: A case report.
}

\author{
Mehrbano Amirshahi, Mahin Badakhsh*, Zohreh Sadat Hashemi \\ Department of Midwifery, School of Nursing and Midwifery, Zabol University of Medical sciences, Zabol, Iran
}

\begin{abstract}
Background: Potter's syndrome is a rare congenital disorder that refers to a set of clinical manifestations that are associated with oligohydramnios caused by fetal kidney failure. The hallmark of this syndrome is the special clinical picture that in addition oligohydramnios characteristic with pulmonary hypoplasia, bilateral renal agenesis; limb deformities and specific face. The embryo dies before or immediately after birth due to respiratory failure. The aim of this study is to report a fetus with Potter's syndrome that was born by vaginal delivery.

Case report: Ultrasound examination of pregnant woman revealed that her male fetus with gestational age of 25 weeks with Potter's syndrome and the amniotic fluid index is zero. The mother was hospitalized in unit labor and delivered in a natural course. The baby had a clinical picture of Potter's syndrome and severe respiratory distress and died shortly after birth.

Conclusion: Potter's syndrome is a very serious condition and most of the time it is deadly. Prenatal ultrasound by examining oligohydramnios and kidneys helps to diagnose.
\end{abstract}

Keywords: Potter's syndrome, Oligohydramnios, Pulmonary hypoplasia, Fetus.

Accepted on February 21, 2019

\section{Introduction}

Potter syndrome is a rare congenital malformation that primarily affects male fetuses and is characterized by pulmonary hypoplasia caused by renal failure. It was first reported by Edith Potter in 1946. After the $16^{\text {th }}$ week of pregnancy, the amount of amniotic fluid depends mainly on the production of urine by the fetus. During the intrauterine life, the fetus continuously swallows the amniotic fluid which returns back to the amniotic sac by the kidneys. Oligohydramnios occur when the volume of amniotic fluid is less than normal for that period of pregnancy. This decrease in volume may be due to a reduction in urine output, secondary to causes such as bilateral renal agenesis, urinary tract obstruction, and prolonged rupture of the amniotic sacs. The fetus urine, essential for the development of lungs, plays its role in contributing to the development of airways and alveoli, creating hydraulic pressure, and providing proline, an essential amino acid for the development of lungs. If the alveoli and as a result the lungs are not adequately developed at birth, the neonate will not be able to breathe well and will suffer from respiratory distress due to pulmonary hypoplasia. Therefore, secondary to renal failure, pulmonary hypoplasia is the main cause of death in neonates with Potter syndrome [1,2].

Production of urine by the embryo not only affects the volume of amniotic fluid, but also preserves the embryo against the mother's uterus wall pressure like a cushion. Oligohydramnios results in a special form of fetus called "Potter facies", which is characterized by features such as flattened nasal bridge, hypoplastic chin, epicanthal folds, cataract, and low-set ears [3].

The main cause of the Potter syndrome is unknown; this syndrome has a genetic background in some cases, and is more common in neonates with a family history of kidney abnormalities [4]. The syndrome, with an incidence of 1 in every 2,000 to 5,000 fetuses, is associated with a recurrence risk of $3-6 \%$, and is found in $0.2-0.4 \%$ of autopsies in dead new-borns or those who die immediately after birth [5]. However, it is believed that the disease may have a higher prevalence because the affected fetuses are born dead or die shortly after the birth. There is no known method for preventing this deadly disease. Therefore, screening ultrasound is recommended at 16-18 weeks of gestation for mothers with a positive history of pregnancy for this syndrome aiming to evaluate oligohydramnios and fetal kidneys. Although this syndrome has deadly consequences and is not compatible with life and ends with the baby's death, the affected fetuses require resuscitation and treatment of urinary output obstruction at the time of delivery [6]. The present study aimed to report a fetus with Potter syndrome that was born through normal delivery.

\section{Case Report}

The case was a 25-week-old male fetus with Potter syndrome, born through normal delivery. The mother was admitted to the maternity ward of Amir al-Momenin Hospital, Zabol, Iran, on May 10, 2017 with an ultrasound, which showed the absence of amniotic fluid and the diagnosis of Potter syndrome for the fetus. The mother was 24 years old, Iranian, housewife, gravid 
3, and para 1, and had an abortion history at Month 1 . She did not remember the first day of her last menstruation, and her gestational age was 25 weeks according to the only ultrasound performed on the same day. She had a normal childbirth history (her previous child was healthy and had normal delivery), and had received prenatal care during pregnancy. Her pregnancy tests were normal. There were no history of smoking, drugs or alcohol, underlying diseases such as diabetes, hypertension, thyroid, cardiopulmonary and renal, infection, and drug use other than routine pregnancy medicines (iron and vitamin supplements). The mother and her husband were cousins and she did not mention a family history of a neonatal birth with a similar disorder. Prenatal serological tests were negative for syphilis, AIDS, hepatitis B, and rubella. She had a blood type B positive.

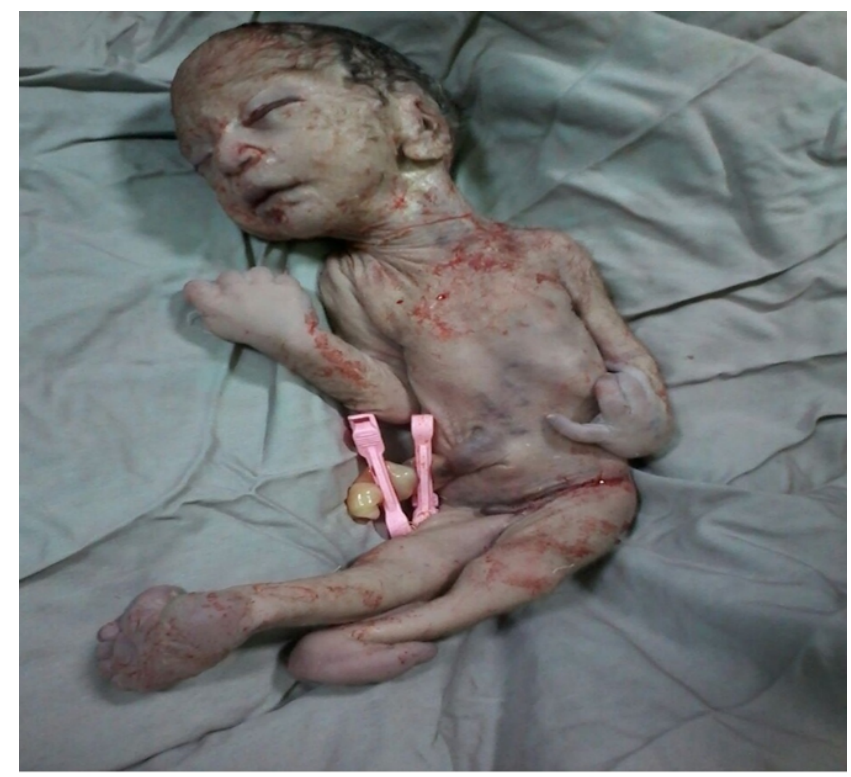

Figure 1. Potter syndrome overall characteristics.

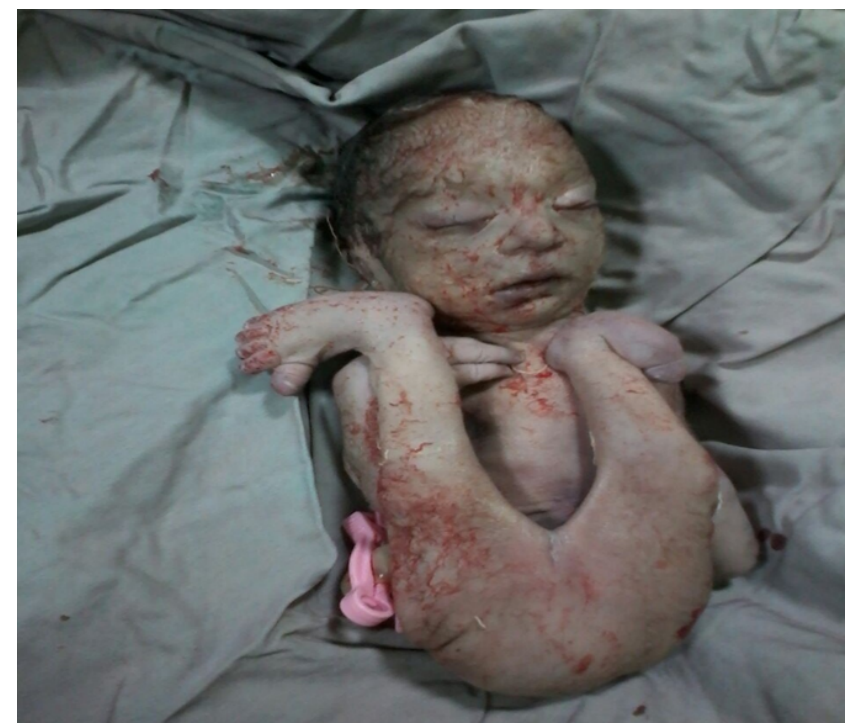

Figure 2. Potter syndrome with imperforate anus.
According to the mentioned ultrasound, the gestational age was 25 weeks, the amniotic fluid index was zero, the fetus kidneys were invisible in the normal anatomical position, the bladder was invisible, and there was severe fetal growth restriction and severe cardiomegaly (more than $80 \%$ ). The diagnosis was Potter syndrome based on ultrasound, after which the mother was taken to the maternity ward.

The mother was hospitalized and, after the normal course of labor, gave birth to a male new-born through vaginal method. The boy had growth limitation ( $800 \mathrm{~g}$ birth weight), the clinical presentation of Potter syndrome, and severe respiratory distress and died immediately after delivery (Figures 1 and 2).

\section{Discussion}

The present study introduced a rare case that has not been reported in the region so far. It was a fatal case of Potter syndrome that was born through normal delivery. The ultrasound of the woman revealed a fetus at 25 weeks of pregnancy with Potter syndrome and an amniotic fluid index of zero. The mother was admitted to the delivery unit and the baby was born vaginally. The new-born with the clinical presentation of Potter syndrome had severe respiratory distress and died shortly after birth.

Potter syndrome, first described by Edith Potter in neonates, is characterized by bilateral renal agenesis or other renal abnormalities such as aplasia, dysplasia, hypoplasia, or polycystic kidney disease. It is recognized more in boys with a male to female ratio of 2 to 1 , suggesting the presence of certain genes on chromosome Y [1]. Potter syndrome can be seen in neonates with normal kidneys, but the mother had a chronic and prolonged amniotic fluid leakage during the middle weeks of pregnancy [7].

In this study, the ultrasound of the pregnant women revealed a case of Potter syndrome in a male fetus in the 25th week of pregnancy, a zero amniotic fluid index, and the lack of both kidneys and bladder. Ultrasound findings of fetuses with severe kidney disease in 23 families indicated prolonged oligohydramnios, severe renal dysfunction, and Potter facies, and the infants died within hours to days after birth. Moreira and Reuvers also pointed out the birth of fetuses with Potter syndrome $[8,9]$.

The neonatal features of Potter syndrome include facial alterations, limb malformations, fetal growth limitation, and pulmonary hypoplasia, known as oligohydramnios tetrad. These features arise from fetal compression due to prolonged oligohydramnios. Potter facies is characterized by hypertelorism, Mongolian eyelid, epicanthic fold, flattened nasal bridge and ear lobe, parrot beak nose, low-set ears, recessed chin, a small fold below the lip, short neck, and extra chains around the neck. The clinical presentation of the infant in this study was recessed face, flattened nasal bridge and ear lobe, parrot beak nose, low-set ears, small, short, and recessed chin, a small fold below the lip short neck, and extra folds around the neck, wide hands, hand deviation at wrist, bilateral valgus, and severe fetal growth limitation, which resulted in a 
birth weight of $800 \mathrm{~g}$. These clinical manifestations were consistent with Shastry and Al-Haggar [10,11].

The constant pressure of the uterine walls on the fetus chest wall and the pressure of intra-abdominal organs on the diaphragm constitute one of the consequences of oligohydramnios in Potter syndrome, which are also the main causes of lung hypoplasia and failure in this syndrome. The severity of the pulmonary hypoplasia depends on the development phase of lung in which oligohydramnios occurs, as well as the intensity and duration of the oligohydramnios. Because of severe respiratory distress and lung hypoplasia, fetuses are born with dysplastic kidneys or die shortly after birth [12]. In this study, the amniotic fluid index was zero according to ultrasonography and the new-born had severe respiratory distress at birth and died a few moments after birth.

Potter syndrome can be associated with congenital heart anomalies, gastrointestinal anomalies (such as esophagus atresia, colonic agenesis, and anal and duodenal malformations, Meckel's diverticulum, and pancreatic and spleen cysts), skeletal disorders, brain abnormalities, and various associations, such as Vakarl et al. [13]. In the present study, the ultrasound reported a severe cardiomegaly (more than $80 \%$ ) and invisible bladder; and the physical examination of the neonate revealed limb anomalies and an imperforate anus.

The main cause of Potter syndrome remains unclear in most cases, but it has a genetic reason in some cases, and the inheritance pattern depends on a particular genetic cause. Genetic abnormalities such as autosomal dominant or recessive inheritance of polycystic kidney disease, hereditary kidney dysplasia, caused by RET and UPK3A gene mutations and chromosomal abnormalities, can result in developmental abnormalities and lead to Potter syndrome. This syndrome occurs sporadically but may be inherited when arising from the autosomal dominant triad. Potter syndrome is more common in infants who have a family history of kidney abnormalities $[4,7]$. No specific cause was found in the present study for this disease, and there was no family history of renal anomalies. San and Samal also pointed out the unknown etiology of Potter syndrome and its genetic reasons in their studies [14,15].

Though the main cause of Potter syndrome is the abnormality in kidneys development, the initial diagnosis is performed by ultrasound that can show the loss or absence of amniotic fluid and the absence of bladder, and is continued through investigation on the presence or absence of kidneys. Genetic counseling is important to confirm diagnosis. The identification of this syndrome, which is characterized by bilateral renal agenesis, pulmonary hypoplasia, Potter facies, and limb malformation, can be problematic by ultrasound due to oligohydramnios [16]. In this study, the ultrasound showed the absence of amniotic fluid index, invisibility of the bladder, and bilateral renal agenesis.

Potter syndrome is incompatible with life and has a deadly prognosis. Pregnancy may be terminated before the fetus reaches the life stage. The standard pregnancy care does not change when it is decided to continue the pregnancy [17]. One of the limitations of the present study was that genetic testing was not performed, since the parents did not accept autopsy.

In the present study, a mother in her $25^{\text {th }}$ week of pregnancy with a Potter syndrome fetus, diagnosed through ultrasound, was admitted to the delivery unit and gave a normal birth to a baby who died a few moments after birth.

\section{Conclusion}

Potter syndrome is a very serious illness and a deadly condition. Prenatal ultrasound helps detect this syndrome by examining oligohydramnios and kidney conditions.

\section{Conflict of Interest}

The authors declare that they have no conflict of interest regarding the publication of this case report.

\section{Funding}

No funding was sought or secured in relation to this case report.

\section{Patient Consent}

Obtained

\section{Provenance and Peer Review}

This case report was peer reviewed.

\section{References}

1. Potter EL. Facial characteristics of infants with bilateral renal agenesis. Am J Obstet Gynecol 1946; 51: 885-888.

2. Srihari Babu M, Asha Latha D, Radhika P. Potters syndrome: A case report. JDMS 2015; 14: 14-16.

3. Sudhanshu Kumar D, Sidharth Sankar M. Potter facies with polycystic kidney disease in association with other congenital anomalies: two case reports. J Med Dent Sci 2013; 2: 2665-2668.

4. Khatami F. Potters syndrome: A study of 15 patients. Arch Iranian Med 2004; 7: 186- 189.

5. Sarkar S, Das Gupta S, Barua M, Ghosh R, Mondal K, Chatterjee U, Datta C. Potters sequence: A story of the rare, rarer and the rarest. Indian J Pathol Microbiol 2015; 58: 102-104.

6. Manoj MG, Kakkar S. Potters syndrome-A fatal constellation of anomalies. Indian J Med Res 2014; 139: 648-649.

7. Himabindu A, Narasinga B. A fatal case of potters syndrome-a case report. JCDR 2011; 5: 1264-1266.

8. Moreira BL, Monarim MA, Romano RF, Mattos LA, DIppolito G. Doege-Potter syndrome. Radiol Bras 2015; 48: $195-196$ 
9. Reuvers JR, van Dorp M, Van Schil PE. Solitary fibrous tumor of the pleura with associated Doege-Potter syndrome. Acta Chir Belg 2016; 116: 386-387.

10. Shastry SM, Kolte SS, Sanagapati PR. Potters sequence. J Clin Neonatol 2012; 1: 157-159.

11. Al-Haggar M, Yahia S, Abdel-Hadi D, Grill F, Al-Kaissi A. Sirenomelia (symelia apus) with Potters syndrome in connection with gestational diabetes mellitus: A case report and literature review. Afr Health Sci 2010; 10: 395-399.

12. Dhundiraj KM, Madhukar DN, Ambadasrao PG, Wamanrao KS, Prem ZM. Potters syndrome: A report of 5 cases. Indian J Pathol Microbiol 2006; 49: 254-257.

13. Dayal J, Maheshwari E, Ghai PS, Bhatotia S. Antenatal ultrasound diagnosis of Potters syndrome. Indian J Radiol Imaging 2003; 13: 81-83.

14. San Agustin JT, Klena N, Granath K, Panigrahy A, Stewart E, Devine W, Strittmatter L, Jonassen JA, Liu X. Genetic link between renal birth defects and congenital heart disease. Nat Commun 2016; 22: 111-118.
15. Samal SK, Rathod S. Sirenomelia: The mermaid syndrome: report of two cases. J Nat Sci Biol Med 2015; 6: 264-266.

16. Gerards FA, Twisk JW, Fetter WP. Predicting pulmonary hypoplasia with 2- or 3-dimensional ultrasonography in complicated pregnancies. Am J Obstet Gynecol 2008; 198: 140-146.

17. Kemper MJ, Mueller-Wiefel DE. Prognosis of antenatally diagnosed oligohydramnios of renal origin. Eur J Pediatr 2007; 166: 393-398.

\section{*Correspondence to}

Mahin Badakhsh

Department of Midwifery

School of Nursing and Midwifery

Zabol University of Medical sciences

Iran 